\title{
An assessment of the Gurson yield criterion by a computational multi-scale approach
}

\author{
S.M. Giusti ${ }^{a}$, P.J. Blanco ${ }^{a}$, E.A. de Souza Neto ${ }^{b, *}$ and \\ R.A. Feijóo ${ }^{a}$ \\ ${ }^{a}$ LNCC, Laboratório Nacional de Computação Científica, Av. Getúlio Vargas 333, \\ Quitandinha,25651-075, Petrópolis, RJ, Brasil,e-mail: giusti@lncc.br, \\ pjblanco@lncc.br,feij@lncc.br \\ ${ }^{\mathrm{b}}$ Civil and Computational Engineering Centre, School of Engineering, University \\ of Wales Swansea, Singleton Park, Swansea SA2 8PP, United Kingdom
}

\begin{abstract}
Purpose Assess the Gurson yield criterion for porous ductile metals.

Methodology A finite element procedure is used within a purely kinematical multiscale constitutive modelling framework to determine estimates of extremal overall yield surfaces. The RVEs analysed consist of an elastic-perfectly plastic von Mises type matrix under plane strain conditions containing a single centered circular hole. Macroscopic yield surface estimates are obtained under three different RVE kinematical assumptions: linear boundary displacements (an upper bound); periodic boundary displacement fluctuations (corresponding to periodically perforated media); and, minimum constraint or uniform boundary traction (a lower bound).

Findings The Gurson criterion predictions fall within the bounds obtained under relatively high void ratios - when the bounds lie farther apart. Under lower void ratios, when the bounds lie close together, the Gurson predictions of yield strength lie slightly above the computed upper bounds in regions of intermediate to high stress triaxiality. A modification to the original Gurson yield function is proposed that can capture the computed estimates under the three RVE kinematical constraints considered.
\end{abstract}

Originality Assesses the accuracy of the Gurson criterion by means of a fully computational multi-scale approach to constitutive modelling. Provides an alternative criterion for porous plastic media which encompasses the common microscopic kinematical constraints adopted in this context.

Keywords Porous plasticity, Gurson model, multi-scale modelling, finite element. Article type Research paper

* Corresponding author.

Email address: e.desouzaneto@swansea.ac.uk (E.A. de Souza Neto). 


\section{Introduction}

Over the past decade, the use of analytical and computational tools for the prediction of the constitutive behaviour of materials relying on information at two or more physical scales has been the subject of increasing interest in academic circles. Particularly interesting applications of multi-scale concepts include: (a) The estimation of the effective parameters of a pre-defined macroscopic continuum constitutive model as well as the definition of new phenomenological macroscopic constitutive models through the analysis of a microscopic representative volume element (RVE) (Hill (1963); Gurson (1977); Michel \& Suquet (1992); Gărăjeu \& Suquet (1997); Michel et al. (1999, 2001); Pellegrino et al. (1997, 1999)), and (b) The development and use of macroscopic constitutive models whose generally dissipative behaviour is the result of the homogenisation of the response of a RVE, without reference to any pre-defined set of constitutive equations at the macroscopic scale (Swan (1994); Miehe et al. (1999, 2002); Kouznetsova et al. (2002); Terada et al. (2003)).

Within the category of applications classed as (a) in the above, the seminal paper by Gurson (1977) deserves special mention. In his paper, Gurson has derived upper bound yield loci estimates for porous ductile metals by means of a semi-analytical method based on the study of the collapse of representative perforated rigid-perfectly plastic cells under an assumed mechanism. This work has been followed up by other researchers who proposed refinements to the original Gurson model (see, for example, Tvergaard (1981)). Further multi-scale based studies of porous plastic media have been conducted, among others, by Gărăjeu \& Suquet (1997) who proposed a generalisation of Gurson's yield function to account for the presence of rigid inclusions within the porous microstructure, and by Michel et al. (2001) who assessed the influence of the randomness of void distribution on the macroscopic yield strength.

Our main purpose in the present paper is to further investigate Gurson's plane strain criterion by assessing it against finite element predictions of extremal yield surfaces for porous plastic media. The numerical predictions are obtained here within a purely kinematical homogenisation-based multi-scale constitutive framework. Square RVEs consisting of an elastic-perfectly plastic von Mises type matrix containing a circular void are considered in the computational homogenisation procedure. The paper is organised as follows. The kinematical multi-scale constitutive framework is reviewed in Section 2. Within this framework, upper and lower bounds of the homogenised constitutive behaviour correspond respectively to the choices of: (i) linear RVE boundary displacements - the most constrained - and, (ii) minimum kinematical constraint - or uniform RVE boundary tractions. A further prediction can be obtained under the kinematical assumption of periodicity of the RVE boundary displacement fluctuation - typically associated with the anal- 


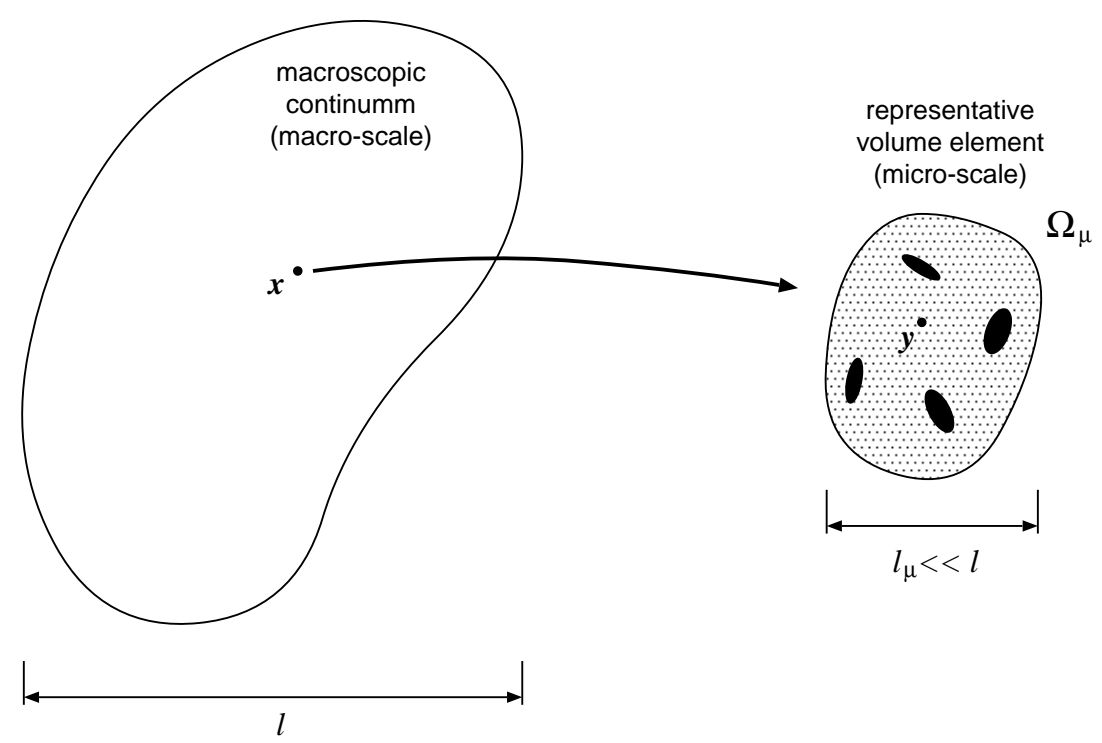

Fig. 1. Macro-continuum with a locally attached micro-structure.

ysis of microstructurally periodic media. The finite element implementation of the resulting multi-scale constitutive models is described in Section 3. Here, the linearised system of algebraic equations required by the Newton-Raphson scheme for the solution of the associated incremental microscopic equilibrium problems is briefly described. The kinematical constraints of the RVE are enforced directly upon the finite element-generated spaces of displacement fluctuations and virtual displacements. The main contribution is presented in Section 4. Predictions of extremal yield surfaces are determined within the computational homogenisation framework. The original Gurson upper bound for porous plastic media under plane strain is assessed against the numerical results. A modification of the original Gurson yield function is then suggested which is able to capture the yield loci of porous metals under the three kinematical assumptions considered. The paper ends in Section 5 where some concluding remarks are presented.

\section{Homogenisation-based multi-scale constitutive theory}

The starting point of the kinematically-based family of multi-scale constitutive theories upon which the present paper relies is the assumption that any material point $\boldsymbol{x}$ of the (macroscopic) continuum is associated to a local Representative Volume Element (RVE) whose domain, $\Omega_{\mu}$ (refer to Fig. 1), has a characteristic length, $l_{\mu}$, much smaller than the characteristic length, $l$, of the macro-continuum. At any instant $t$, the strain tensor at an arbitrary point $\boldsymbol{x}$ of the macro-continuum is assumed (de Souza Neto \& Feijóo , 2006) to be the 
volume average of the microscopic strain tensor field, $\varepsilon_{\mu}$, defined over $\Omega_{\mu}$ :

$$
\varepsilon(t)=\frac{1}{V_{\mu}} \int_{\Omega_{\mu}} \varepsilon_{\mu}(\boldsymbol{y}, t) \mathrm{d} V,
$$

where $V_{\mu}$ is the volume of the RVE and

$$
\varepsilon_{\mu}=\nabla^{s} \boldsymbol{u}_{\mu}
$$

where $\nabla^{s} \boldsymbol{u}_{\mu}$ denotes the symmetric gradient of the microscopic displacement field $\boldsymbol{u}_{\mu}$ of the RVE.

\subsection{Kinematically admissible RVE displacement fields}

By replacing (2) into (1) and making use of Green's theorem, it can easily be established that the averaging relation (1) is equivalent to the following constraint on the displacement field of the RVE (de Souza Neto \& Feijóo , 2006):

$$
\int_{\partial \Omega_{\mu}} \boldsymbol{u}_{\mu} \otimes_{s} \boldsymbol{n} \mathrm{d} A=V_{\mu} \boldsymbol{\varepsilon}
$$

where $\boldsymbol{n}$ denotes the outward unit normal field on $\partial \Omega_{\mu}$ and

$$
\boldsymbol{a} \otimes_{s} \boldsymbol{b} \equiv \frac{1}{2}(\boldsymbol{a} \otimes \boldsymbol{b}+\boldsymbol{b} \otimes \boldsymbol{a})
$$

for any vectors $\boldsymbol{a}$ and $\boldsymbol{b}$. This constraint requires the (as yet not defined) set $\mathscr{K}_{\mu}$ of kinematically admissible RVE displacement fields to be a subset of the minimally constrained set of kinematically admissible microscopic displacements, $\mathscr{K}_{\mu}^{*}:$

$$
\mathscr{K}_{\mu} \subset \mathscr{K}_{\mu}^{*} \equiv\left\{\boldsymbol{v}, \text { sufficiently regular } \mid \int_{\partial \Omega_{\mu}} \boldsymbol{v} \otimes_{s} \boldsymbol{n} \mathrm{d} A=V_{\mu} \varepsilon\right\},
$$

with sufficiently regular meaning that the relevant functions have the sufficient degree of regularity so that all operations in which they are involved make sense. By splitting $\boldsymbol{u}_{\mu}$ into a sum

$$
\boldsymbol{u}_{\mu}(\boldsymbol{y}, t)=\boldsymbol{\varepsilon}(t) \boldsymbol{y}+\tilde{\boldsymbol{u}}_{\mu}(\boldsymbol{y}, t)
$$

of a homogeneous strain displacement, $\boldsymbol{\varepsilon}(t) \boldsymbol{y}$, and a displacement fluctuation field, $\tilde{\boldsymbol{u}}_{\mu}$, the above constraint is made equivalent to requiring that the space $\tilde{\mathscr{K}}_{\mu}$ of kinematically admissible displacement fluctuations of the RVE be a subspace of the of the minimally constrained space of kinematically admissible displacement fluctuations, $\tilde{\mathscr{K}}_{\mu}^{*}$ :

$$
\tilde{\mathscr{K}}_{\mu} \subset \tilde{\mathscr{K}}_{\mu}^{*} \equiv\left\{\boldsymbol{v}, \text { sufficiently regular } \mid \int_{\partial \Omega_{\mu}} \boldsymbol{v} \otimes_{s} \boldsymbol{n} \mathrm{d} A=\mathbf{0}\right\} .
$$


Further, it can be trivially established (refer to de Souza Neto \& Feijóo (2006) for details) that the (yet to be defined) space $\tilde{\mathscr{K}}_{\mu}$ coincides with the space of virtual displacements of the RVE.

Following the split (6) the microscopic strain (2) can be expressed as the sum

$$
\varepsilon_{\mu}(\boldsymbol{y}, t)=\varepsilon(t)+\nabla^{s} \tilde{\boldsymbol{u}}_{\mu}(\boldsymbol{y}, t),
$$

of a homogeneous strain field (coinciding with the macroscopic, average strain) and a field $\nabla^{s} \tilde{\boldsymbol{u}}_{\mu}$ that represents a fluctuation about the average.

\subsection{Macroscopic stress, Hill-Mandel Principle and RVE equilibrium}

Similarly to the macroscopic strain definition (2), the macroscopic stress tensor, $\boldsymbol{\sigma}$, is defined as the volume average of the microscopic stress field, $\boldsymbol{\sigma}_{\mu}$, over the RVE:

$$
\boldsymbol{\sigma}(t) \equiv \frac{1}{V_{\mu}} \int_{\Omega_{\mu}} \boldsymbol{\sigma}_{\mu}(\boldsymbol{y}, t) \mathrm{d} V
$$

Another crucial concept underlying models of the present type is the HillMandel Principle of Macro-homogeneity (Hill , 1965; Mandel , 1971) which requires the macroscopic stress power to equal the volume average of the microscopic stress power for any kinematically admissible motion of the RVE. This is expressed by the equation

$$
\boldsymbol{\sigma}: \dot{\varepsilon}=\frac{1}{V_{\mu}} \int_{\Omega_{\mu}} \boldsymbol{\sigma}_{\mu}: \dot{\varepsilon}_{\mu} \mathrm{d} V
$$

that must hold for any kinematically admissible microscopic strain rate field, $\dot{\varepsilon}_{\mu}$. The above is equivalent to the following variational equation:

$$
\int_{\partial \Omega_{\mu}} \boldsymbol{t} \cdot \boldsymbol{\eta} \mathrm{d} A=0 ; \quad \int_{\Omega_{\mu}} \boldsymbol{b} \cdot \boldsymbol{\eta} \mathrm{d} V=0 \quad \forall \boldsymbol{\eta} \in \tilde{\mathscr{K}}_{\mu}
$$

in terms of the RVE boundary traction and body force fields denoted, respectively, $\boldsymbol{t}$ and $\boldsymbol{b}$. That is, the virtual work of the RVE body force and surface traction fields vanish - they are the reaction forces associated to the imposed kinematical constraints embedded in the choice of $\tilde{\mathscr{K}}_{\mu}$.

With the above at hand, the variational equilibrium statement - the virtual work equation - for the RVE is given by

$$
\int_{\Omega_{\mu}} \boldsymbol{\sigma}_{\mu}: \nabla^{s} \boldsymbol{\eta} \mathrm{d} V=0 \quad \forall \boldsymbol{\eta} \in \tilde{\mathscr{K}}_{\mu}
$$


Further, we assume that at any time $t$ the stress at each point $\boldsymbol{y}$ of the RVE is delivered by a generic constitutive functional $\mathfrak{S}_{y}$ of the strain history $\varepsilon_{\mu}^{t}(\boldsymbol{y})$ at that point up to time $t$ :

$$
\boldsymbol{\sigma}(\boldsymbol{y}, t)=\mathfrak{S}_{y}\left(\varepsilon_{\mu}^{t}(\boldsymbol{y})\right)
$$

This constitutive assumption, together with the equilibrium equation (12) leads to the definition of the RVE equilibrium problem which consists in finding, for a given macroscopic strain $\varepsilon$ (a function of time), a displacement fluctuation function $\tilde{\boldsymbol{u}}_{\mu} \in \tilde{\mathscr{K}}_{\mu}$ such that

$$
\int_{\Omega_{\mu}^{s}} \mathfrak{S}_{y}\left\{\left[\boldsymbol{\varepsilon}(t)+\nabla^{s} \tilde{\boldsymbol{u}}_{\mu}(\boldsymbol{y}, t)\right]^{t}\right\}: \nabla^{s} \boldsymbol{\eta} \mathrm{d} V=0 \quad \forall \boldsymbol{\eta} \in \tilde{\mathscr{K}}_{\mu}
$$

\subsection{Characterisation of the multi-scale constitutive model}

The general multi-scale constitutive model in the present context is defined as follows. For a given macroscopic strain history, we must firstly solve the RVE equilibrium problem defined by (14). With the solution $\tilde{\boldsymbol{u}}_{\mu}$ at hand, the macroscopic stress tensor is determined according to the averaging relation (9), i.e., we have

$$
\boldsymbol{\sigma}(t)=\mathfrak{S}\left(\varepsilon^{t}\right) \equiv \frac{1}{V_{\mu}} \int_{\Omega_{\mu}} \mathfrak{S}_{y}\left\{\left[\varepsilon+\nabla^{s} \tilde{\boldsymbol{u}}_{\mu}\right]^{t}\right\} \mathrm{d} V
$$

where $\mathfrak{S}$ denotes the resulting (homogenised) macroscopic constitutive functional.

\subsubsection{The choice of kinematical constraints}

The characterisation of a multi-scale model of the present type is completed with the choice of a suitable space of kinematically admissible displacement fluctuations, $\tilde{\mathscr{K}}_{\mu} \subset \tilde{\mathscr{K}}_{\mu}^{*}$. In general, different choices lead to different macroscopic response functionals. The following choices will be considered in the assessment of the Gurson model addressed in Section 4:

(i) Linear boundary displacements (or zero boundary fluctuations) model:

$$
\tilde{\mathscr{K}}_{\mu}=\tilde{\mathscr{K}}_{\text {lin }} \equiv\left\{\boldsymbol{v}, \text { sufficiently regular } \mid \boldsymbol{v}(\boldsymbol{y})=\mathbf{0} \forall \boldsymbol{y} \in \partial \Omega_{\mu}\right\} .
$$

The displacements of the boundary of the RVE for this class of models are fully prescribed as

$$
\boldsymbol{u}_{\mu}(\boldsymbol{y})=\boldsymbol{\varepsilon} \boldsymbol{y} \quad \forall \boldsymbol{y} \in \partial \Omega_{\mu}
$$



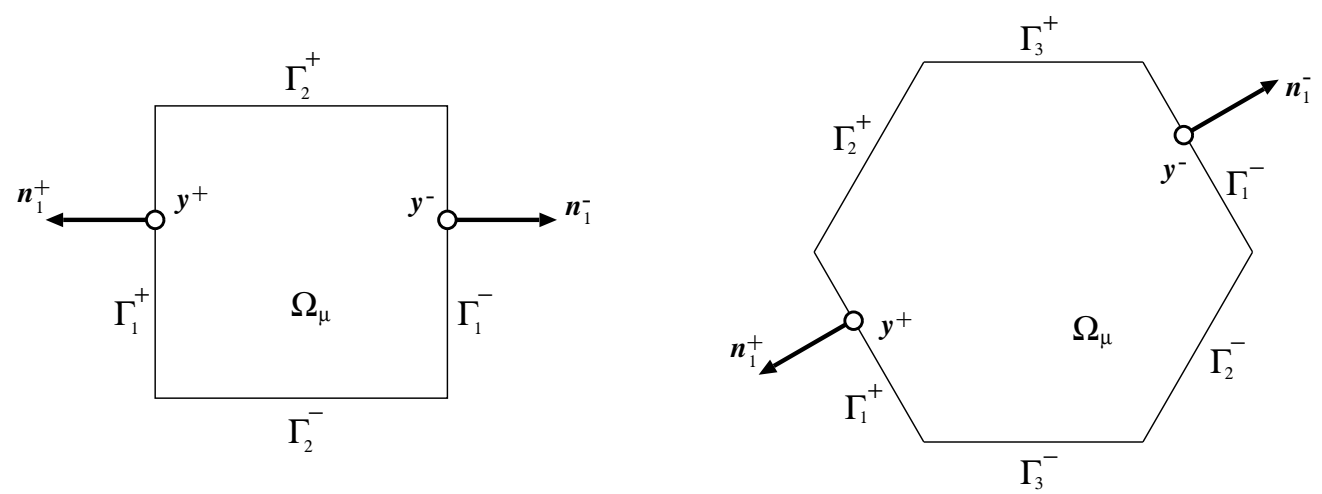

Fig. 2. RVE geometries for periodic media. Square and hexagonal cells.

(ii) Periodic boundary fluctuations. This assumption is typically associated with the description of media with periodic microstructure. The macrostructure in this case is generated by the periodic repetition of the RVE (Michel et al. , 1999). For simplicity, we will focus the description on two-dimensional problems and we shall follow the notation adopted by Miehe et al. (1999). Consider, for example, the square or hexagonal RVEs, as illustrated in Figure 2. In this case, each pair $i$ of sides consists of equally sized subsets

$$
\Gamma_{i}^{+} \text {and } \Gamma_{i}^{-}
$$

of $\partial \Omega_{\mu}$, with respective unit normals

$$
\boldsymbol{n}_{i}^{+} \text {and } \boldsymbol{n}_{i}^{-}
$$

such that

$$
\boldsymbol{n}_{i}^{-}=-\boldsymbol{n}_{i}^{+}
$$

A one-to-one correspondence exists between the points of $\Gamma_{i}^{+}$and $\Gamma_{i}^{-}$. That is, each point $\boldsymbol{y}^{+} \in \Gamma_{i}^{+}$has a corresponding pair $\boldsymbol{y}^{-} \in \Gamma_{i}^{-}$.

The key kinematical constraint for this class of models is that the displacement fluctuation must be periodic on the boundary of the RVE. That is, for each pair $\left\{\boldsymbol{y}^{+}, \boldsymbol{y}^{-}\right\}$of boundary material points we have

$$
\tilde{\boldsymbol{u}}_{\mu}\left(\boldsymbol{y}^{+}, t\right)=\tilde{\boldsymbol{u}}_{\mu}\left(\boldsymbol{y}^{-}, t\right) .
$$

Accordingly, the space $\tilde{\mathscr{K}}_{\mu}$ is defined as

$$
\tilde{\mathscr{K}}_{\mu}=\tilde{\mathscr{K}}_{\text {per }} \equiv\left\{\tilde{\boldsymbol{u}}_{\mu}, \text { suff. reg. } \mid \tilde{\boldsymbol{u}}_{\mu}\left(\boldsymbol{y}^{+}, t\right)=\tilde{\boldsymbol{u}}_{\mu}\left(\boldsymbol{y}^{-}, t\right) \quad \forall \text { pairs }\left\{\boldsymbol{y}^{+}, \boldsymbol{y}^{-}\right\}\right\} .
$$

(iii) The minimally constrained (or uniform boundary traction) model:

$$
\tilde{\mathscr{K}}_{\mu} \equiv \tilde{\mathscr{K}}_{\mu}^{*}
$$

It can be shown (de Souza Neto \& Feijóo , 2006) that the distribution of stress vector on the RVE boundary, reactive to the minimal kinematic 
constraint, satisfies

$$
\boldsymbol{\sigma}_{\mu}(\boldsymbol{y}, t) \boldsymbol{n}(\boldsymbol{y})=\boldsymbol{\sigma}(t) \boldsymbol{n}(\boldsymbol{y}) \quad \forall \boldsymbol{y} \in \partial \Omega_{\mu}
$$

As for the linear boundary displacements assumption, there are no restrictions on the geometry of the RVE in the present case.

\section{Finite element approximation}

This section provides a brief description of the computational implementation of multi-scale constitutive theories of the above type within a non-linear finite element framework. At the outset, we shall assume the constitutive behaviour at the RVE level to be described by conventional internal variable-based dissipative constitutive theories, whereby the stress tensor is obtained by integrating a set of ordinary differential equations in time (or pseudo-time) for the given strain tensor history. Elasto-plasticity and visco-plasticity are classical, widely used examples of such specialisations of (13). In these cases, numerical approximations to the initial value problem defined by the constitutive equations of the model are usually obtained by Euler-type difference schemes. For a typical time (or pseudo-time) interval $\left[t^{n}, t^{n+1}\right]$, with known set $\boldsymbol{\alpha}^{n}$ of internal variables at $t^{n}$, the stress $\boldsymbol{\sigma}_{\mu}^{n+1}$ at $t^{n+1}$ is a (generally implicit) function of the (prescribed) strain $\varepsilon_{\mu}^{n+1}$ at $t^{n+1}$ (refer, for instance, to Simo \& Hughes (1998) for a detailed account of procedures of this kind in the context of plasticity and visco-plasticity). This can be symbolically represented as

$$
\boldsymbol{\sigma}_{\mu}^{n+1}=\hat{\boldsymbol{\sigma}}_{y}\left(\varepsilon_{\mu}^{n+1} ; \boldsymbol{\alpha}^{n}\right)
$$

where $\hat{\boldsymbol{\sigma}}_{y}$ denotes the integration algorithm-related implicit incremental constitutive function at the point of interest, $\boldsymbol{y}$.

The above leads to the definition of an incremental version of the homogenised constitutive function defined in (15), obtained by replacing $\mathfrak{S}_{y}$ with its timediscrete counterpart $\hat{\boldsymbol{\sigma}}_{y}$ :

$$
\boldsymbol{\sigma}^{n+1}=\hat{\boldsymbol{\sigma}}\left(\varepsilon^{n+1} ; \overline{\boldsymbol{\alpha}}^{n}\right) \equiv \frac{1}{V_{\mu}} \int_{\Omega_{\mu}} \hat{\boldsymbol{\sigma}}_{y}\left(\boldsymbol{\varepsilon}^{n+1}+\nabla^{s} \tilde{\boldsymbol{u}}_{\mu}^{n+1} ; \boldsymbol{\alpha}^{n}\right) \mathrm{d} V
$$

where $\overline{\boldsymbol{\alpha}}^{n}$ denotes the field of internal variable sets over $\Omega_{\mu}$ at time $t^{n}$ and $\tilde{\boldsymbol{u}}_{\mu}^{n+1}$ is the displacement fluctuation field of the RVE at $t^{n+1}-$ the solution to the time-discrete version of equilibrium problem (14):

$$
\int_{\Omega_{\mu}^{s}} \hat{\boldsymbol{\sigma}}_{y}\left(\varepsilon^{n+1}+\nabla^{s} \tilde{\boldsymbol{u}}_{\mu}^{n+1} ; \boldsymbol{\alpha}^{n}\right): \nabla^{s} \boldsymbol{\eta} \mathrm{d} V=0 \quad \forall \boldsymbol{\eta} \in \tilde{\mathscr{K}}_{\mu}
$$




\subsection{Finite element discretisation and solution}

We now focus on the finite element solution of the time-discrete equilibrium problem (25) - a crucial step in the definition of the approximate homogenised constitutive functional. Following a standard notation, the finite element approximation to problem (25) for a given discretisation $h$ consists in determining the unknown vector $\tilde{\mathbf{u}}_{\mu}^{n+1} \in \tilde{\mathscr{K}}_{\mu}^{h}$ of global nodal displacement fluctuations such that

$$
\begin{aligned}
& G^{h}\left(\tilde{\mathbf{u}}_{\mu}^{n+1}\right) \equiv\left\{\int_{\Omega_{\mu}^{h}} \mathbf{B}^{T} \hat{\boldsymbol{\sigma}}_{y}\left(\varepsilon^{n+1}+\mathbf{B} \tilde{\mathbf{u}}_{\mu}^{n+1}\right) \mathrm{d} V\right\} \cdot \boldsymbol{\eta}=0 \\
& \forall \eta \in \tilde{\mathscr{K}}_{\mu}^{h}
\end{aligned}
$$

where $\Omega_{\mu}^{h}$ denotes the discretised RVE domain, $\mathbf{B}$ the global strain-displacement matrix (or discrete symmetric gradient operator), $\varepsilon^{n+1}$ is the fixed (given) array of macroscopic engineering strains at $t_{n+1}, \hat{\boldsymbol{\sigma}}_{y}$ (with upright $\boldsymbol{\sigma}$ ) is the functional that delivers the finite element array of stress components, $\boldsymbol{\eta}$ denotes global vectors of nodal virtual displacements of the RVE and $\tilde{\mathscr{K}}_{\mu}^{h}$ is the finite-dimensional space of virtual nodal displacement vectors associated with the finite element discretisation $h$ of the domain $\Omega_{\mu}$.

The solution to the (generally non-linear) problem (26) can be efficiently undertaken by the Newton-Raphson iterative scheme, whose typical iteration $(k)$ consists in solving the linearised form,

$$
\left[\mathbf{F}^{(k-1)}+\mathbf{K}^{(k-1)} \delta \tilde{\mathbf{u}}_{\mu}^{(k)}\right] \cdot \boldsymbol{\eta}=0 \quad \forall \boldsymbol{\eta} \in \tilde{\mathscr{K}}_{\mu}^{h},
$$

for the unknown iterative nodal displacement fluctuations vector, $\delta \tilde{\mathbf{u}}_{\mu}^{(k)} \in \tilde{\mathscr{K}}_{\mu}^{h}$ where

$$
\mathbf{F}^{(k-1)} \equiv \int_{\Omega_{\mu}^{h}} \mathbf{B}^{T} \hat{\boldsymbol{\sigma}}_{y}\left(\varepsilon^{n+1}+\mathbf{B} \tilde{\mathbf{u}}_{\mu}^{(k-1)}\right) \mathrm{d} V
$$

and

$$
\mathbf{K}^{(k-1)} \equiv \int_{\Omega_{\mu}^{h}} \mathbf{B}^{T} \mathbf{D}^{(k-1)} \mathbf{B} \mathrm{d} V
$$

is the tangent stiffness matrix of the RVE with

$$
\left.\mathbf{D}^{(k-1)} \equiv \frac{\mathrm{d} \hat{\boldsymbol{\sigma}}_{y}}{\mathrm{~d} \varepsilon}\right|_{\mathcal{\varepsilon}=\varepsilon^{n+1}+\mathbf{B} \tilde{\mathbf{u}}_{\mu}^{(k-1)}}
$$

denoting the consistent constitutive tangent matrix field over the RVE domain. In the above the bracketed superscript denotes the Newton iteration number and the time station superscript $n+1$ has been dropped whenever convenient for ease of notational. With the solution $\delta \tilde{\mathbf{u}}_{\mu}^{(k)}$ at hand, the new guess $\tilde{\mathbf{u}}_{\mu}^{(k)}$ for the displacement fluctuation at $t_{n+1}$ is obtained according to the Newton- 

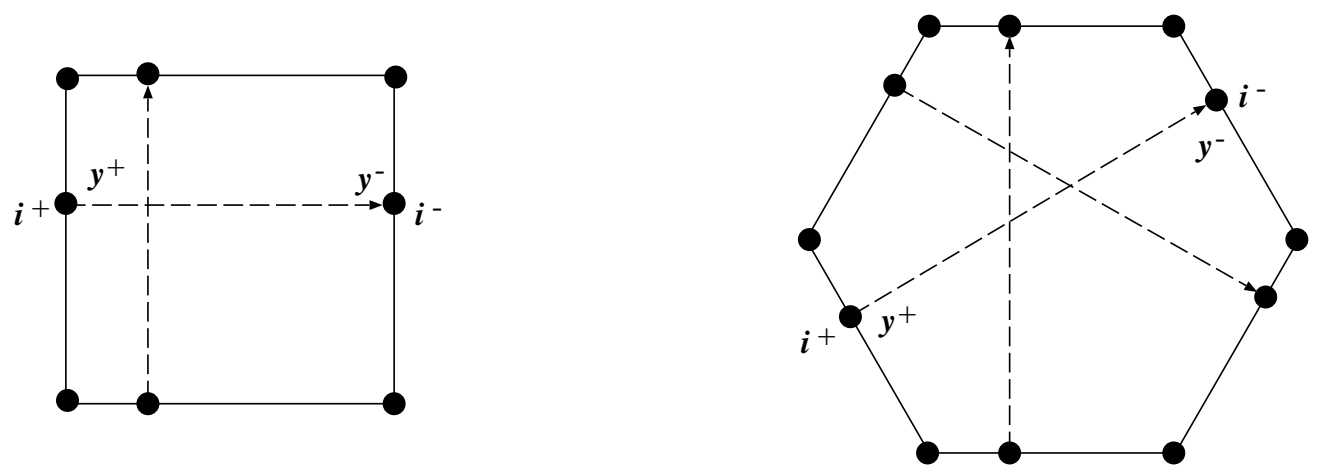

Fig. 3. Discretised RVEs for periodic media.

Raphson update formula

$$
\tilde{\mathbf{u}}_{\mu}^{(k)}=\tilde{\mathbf{u}}_{\mu}^{(k-1)}+\delta \tilde{\mathbf{u}}_{\mu}^{(k)}
$$

Under the assumption of linear boundary displacements, the solution of problem (27) follows the conventional route of general linear solid mechanics problems - here with the degrees of freedom (fluctuations) of the boundary fully prescribed as zero. Hence, the finite element implementation of this class of multi-scale models requires no further consideration. For the periodic boundary condition and minimally constrained models, however, the kinematic boundary conditions of the RVE are non-conventional. The main difference lies in the finite element-generated finite dimensional spaces of admissible fluctuations and virtual displacements whose constraints, here, are not simply described in terms of either fully constrained or completely free nodal degrees of freedom. For the sake of completeness, this issue is addressed in the following.

\subsubsection{Periodic boundary fluctuations model}

For the periodic boundary displacement fluctuations model, the RVE geometry must comply with the constraints set out in item (iii) listed in Section 2.3.1. In this case, it is convenient to assume, ${ }^{1}$ further, that each boundary node $i^{+}$, with coordinates $\boldsymbol{y}_{i}^{+}$, has a pair $i^{-}$, with coordinates $\boldsymbol{y}_{i}^{-}$, as schematically illustrated in Figure 3. Under this assumption, the space $\tilde{\mathscr{K}}_{\mu}^{h}$ of discretised kinematically admissible nodal displacement fluctuation vectors (with period-

\footnotetext{
$\overline{1}$ This assumption is not necessary, but simplifies considerably the finite element implementation of the periodic fluctuations model.
} 
icity on the boundary) can then be defined as

$$
\tilde{\mathscr{K}}_{\mu}^{h}=\left\{\mathbf{v}=\left[\begin{array}{c}
\mathbf{v}_{i} \\
\mathbf{v}_{+} \\
\mathbf{v}_{-}
\end{array}\right] \quad \mid \mathbf{v}_{+}=\mathbf{v}_{-}\right\}
$$

where $\mathbf{v}_{i}, \mathbf{v}_{+}$and $\mathbf{v}_{-}$denote the vectors containing, respectively, the degrees of freedom of the RVE interior and the portions $\Gamma_{+}$and $\Gamma_{-}$of the RVE boundary. Here we adopt the direct approach suggested by Michel et al. (1999) whereby the periodicity constraint is enforced exactly in the discretised space of fluctuations and virtual displacements. This is at variance with the treatment adopted by Miehe \& Koch (2002) who used a Lagrange multiplier method to enforce the discrete space constraint.

By splitting $\mathbf{F}, \mathbf{K}, \delta \tilde{\mathbf{u}}_{\mu}$ and $\boldsymbol{\eta}$ in the same fashion as $\mathbf{v}$ in the above and taking into account definition (32) as well as the fact that both $\boldsymbol{\eta}$ and $\delta \tilde{\mathbf{u}}_{\mu}^{(k)}$ belong to space $\tilde{\mathscr{K}}_{\mu}^{h}$, the linearised equation (27) takes the form

$$
\left\{\left[\begin{array}{l}
\mathbf{F}_{i} \\
\mathbf{F}_{+} \\
\mathbf{F}_{-}
\end{array}\right]^{(k-1)}+\left[\begin{array}{ccc}
\mathbf{k}_{i i} & \mathbf{k}_{i+} & \mathbf{k}_{i-} \\
\mathbf{k}_{+i} & \mathbf{k}_{++} & \mathbf{k}_{+-} \\
\mathbf{k}_{-i} & \mathbf{k}_{-+} & \mathbf{k}_{--}
\end{array}\right]^{(k-1)}\left[\begin{array}{c}
\delta \tilde{\mathbf{u}}_{i} \\
\delta \tilde{\mathbf{u}}_{+}^{(k)} \\
\delta \tilde{\mathbf{u}}_{+}
\end{array}\right]^{(k)}\right\} \cdot\left[\begin{array}{c}
\boldsymbol{\eta}_{i} \\
\boldsymbol{\eta}_{+} \\
\boldsymbol{\eta}_{+}
\end{array}\right]=0 \quad \forall \boldsymbol{\eta}_{i}, \boldsymbol{\eta}_{+} \cdot
$$

Straightfoward manipulations, considering the repetition of $\delta \tilde{\mathbf{u}}_{+}$and $\boldsymbol{\eta}_{+}$in the relevant vectors of nodal degrees of freedom, reduce the linearised discrete equilibrium equation (33) to the following form

$$
\left\{\begin{array}{r}
\left.\left[\begin{array}{c}
\mathbf{F}_{i} \\
\mathbf{F}_{+}+\mathbf{F}_{-}
\end{array}\right]^{(k-1)}+\left[\begin{array}{cc}
\mathbf{k}_{i i} & \mathbf{k}_{i+}+\mathbf{k}_{i-} \\
\mathbf{k}_{+i}+\mathbf{k}_{-i} & \mathbf{k}_{++}+\mathbf{k}_{+-}+\mathbf{k}_{-+}+\mathbf{k}_{--}
\end{array}\right]^{(k-1)}\left[\begin{array}{c}
\delta \tilde{\mathbf{u}}_{i} \\
\delta \tilde{\mathbf{u}}_{+}
\end{array}\right]^{(k)}\right\} \cdot\left[\begin{array}{c}
\boldsymbol{\eta}_{i} \\
\boldsymbol{\eta}_{+}
\end{array}\right]=0 \\
\forall \boldsymbol{\eta}_{i}, \boldsymbol{\eta}_{+},
\end{array}\right.
$$

which, finally, in view of the arbitrariness of $\boldsymbol{\eta}_{i}$ and $\boldsymbol{\eta}_{+}$, yields the linear system of algebraic equations for the unknown vectors $\delta \tilde{\mathbf{u}}_{i}^{(k)}$ and $\delta \tilde{\mathbf{u}}_{+}^{(k)}$,

$$
\left[\begin{array}{cc}
\mathbf{k}_{i i} & \mathbf{k}_{i+}+\mathbf{k}_{i-} \\
\mathbf{k}_{+i}+\mathbf{k}_{-i} & \mathbf{k}_{++}+\mathbf{k}_{+-}+\mathbf{k}_{-+}+\mathbf{k}_{--}
\end{array}\right]^{(k-1)}\left[\begin{array}{c}
\delta \tilde{\mathbf{u}}_{i} \\
\delta \tilde{\mathbf{u}}_{+}
\end{array}\right]^{(k)}=-\left[\begin{array}{c}
\mathbf{F}_{i} \\
\mathbf{F}_{+}+\mathbf{F}_{-}
\end{array}\right]^{(k-1)} .
$$




\subsubsection{Minimally constrained model}

A procedure completely analogous to the one above is followed to obtain the final Newton-Raphson set of algebraic finite element equations under the assumption of minimally constrained kinematics (or uniform RVE boundary traction). We then start by defining the discrete counterpart of the minimally constrained space of fluctuations and virtual displacements $(7,21)$ :

$$
\tilde{\mathscr{K}}_{\mu}^{h} \equiv\left\{\mathbf{v}=\left[\begin{array}{c}
\mathbf{v}_{i} \\
\mathbf{v}_{b}
\end{array}\right] \mid \int_{\partial \Omega_{\mu}^{h}} \mathbf{N}_{b} \mathbf{v}_{b} \otimes_{s} \boldsymbol{n} \mathrm{d} A=\mathbf{0}\right\},
$$

where $\mathbf{v}_{b}$ is the vector containing the boundary degrees of freedom and $\mathbf{N}_{b}$ is the global interpolation matrix associated solely with the boundary nodes of the discretised RVE.

It can be easily established that the integral constraint on $\mathbf{v}_{b}$ can be equivalently written in matrix form as

$$
\mathrm{Cv}_{b}=\mathbf{0}
$$

where $\mathbf{C}$ is the constraint matrix. For a RVE mesh with $k$ interior nodes and $m$ boundary nodes, in the two-dimensional case $\mathbf{v}_{b}$ is a vector of dimension $2 m$ and $\mathbf{C}$ is the $3 \times 2 m$ matrix given by

$$
\mathbf{C}=\left[\begin{array}{ccccc}
\int_{\partial \Omega_{\mu}^{h}} N_{k+1} n_{1} \mathrm{~d} A & 0 & \cdots & \int_{\partial \Omega_{\mu}^{h}} N_{k+m} n_{1} \mathrm{~d} A & 0 \\
0 & \int_{\partial \Omega_{\mu}^{h}} N_{k+1} n_{2} \mathrm{~d} A & \cdots & 0 & \int_{\partial \Omega_{\mu}^{h}} N_{k+m} n_{2} \mathrm{~d} A \\
\int_{\partial \Omega_{\mu}^{h}} N_{k+1} n_{2} \mathrm{~d} A & \int_{\partial \Omega_{\mu}^{h}} N_{k+1} n_{1} \mathrm{~d} A & \cdots & \int_{\partial \Omega_{\mu}^{h}} N_{m} n_{2} \mathrm{~d} A & \int_{\partial \Omega_{\mu}^{h}} N_{m} n_{1} \mathrm{~d} A
\end{array}\right],
$$

where $n_{1}$ and $n_{2}$ denote the components of the outward unit normal field along the global orthonormal basis $\left\{\boldsymbol{e}_{1}, \boldsymbol{e}_{2}\right\}$ and $N_{j}, j=1, \cdots, m$, are the global shape functions associated with the boundary nodes. In this case, equation (37) poses three linear constraints upon the total number of $2 m$ boundary degrees of freedom of the discrete RVE. For three-dimensional RVEs, $\mathbf{v}_{b}$ is of dimension $3 m$ and matrix $\mathbf{C}$ has dimension $6 \times 3 m$.

In practice, rather than using global shape functions, matrix $\mathbf{C}$ is obtained by assembling elemental matrices which in two dimensions, for an element $e$ with $p$ nodes on the intersection $\Gamma^{(e)}$ between the boundary of the element and the boundary of the RVE, read

$$
\mathbf{C}^{(e)}=\left[\begin{array}{ccccc}
\int_{\Gamma(e)} N_{1}^{(e)} n_{1} \mathrm{~d} A & 0 & \cdots & \int_{\Gamma(e)} N_{p}^{(e)} n_{1} \mathrm{~d} A & 0 \\
0 & \int_{\Gamma(e)} N_{1}^{(e)} n_{2} \mathrm{~d} A & \cdots & 0 & \int_{\Gamma(e)} N_{p}^{(e)} n_{2} \mathrm{~d} A \\
\int_{\Gamma(e)} N_{1}^{(e)} n_{2} \mathrm{~d} A & \int_{\Gamma(e)} N_{1}^{(e)} n_{1} \mathrm{~d} A & \cdots & \int_{\Gamma(e)} N_{p}^{(e)} n_{2} \mathrm{~d} A & \int_{\Gamma(e)} N_{p}^{(e)} n_{1} \mathrm{~d} A
\end{array}\right],
$$

where we have assumed that the nodes of element $e$ lying on $\Gamma^{(e)}$ are locally 
numbered 1 to $p$ and $N_{j}^{(e)}, j=1, \cdots, p$, are the associated local shape functions. For example, a conventional eight-noded bilinear quadrilateral element (of the type employed in Section 4), having a single straight side of length $l^{(e)}$ with $\boldsymbol{n}=\boldsymbol{e}_{1}$ and three equally spaced nodes intersecting the RVE boundary, has

$$
\mathbf{C}^{(e)}=l^{(e)}\left[\begin{array}{cccccc}
\frac{1}{6} & 0 & \frac{2}{3} & 0 & \frac{1}{6} & 0 \\
0 & 0 & 0 & 0 & 0 & 0 \\
0 & \frac{1}{6} & 0 & \frac{2}{3} & 0 & \frac{1}{6}
\end{array}\right] .
$$

In order to handle constraint (37) upon the discrete space of fluctuations and virtual displacements it is convenient to split $\mathbf{v}_{b}$ as

$$
\mathbf{v}_{b}=\left[\begin{array}{c}
\mathbf{v}_{f} \\
\mathbf{v}_{d} \\
\mathbf{v}_{p}
\end{array}\right]
$$

where the subscripts $f, d$ and $p$ stand, respectively, for free, dependent and prescribed degrees of freedom on the boundary of the discrete RVE. Accordingly, the global constraint matrix is partitioned as

$$
\mathbf{C}=\left[\mathbf{C}_{f} \mathbf{C}_{d} \mathbf{C}_{p}\right]
$$

so that the constraint equation (37) reads

$$
\left[\begin{array}{lll}
\mathbf{C}_{f} & \mathbf{C}_{d} & \mathbf{C}_{p}
\end{array}\right]\left[\begin{array}{c}
\mathbf{v}_{f} \\
\mathbf{v}_{d} \\
\mathbf{v}_{p}
\end{array}\right]=\mathbf{0}
$$

Prescribed degrees of freedom are needed here in order to remove rigid body displacements of the RVE and make the corresponding discrete equilibrium problem (26) well-posed. Trivially, we then prescribe

$$
\mathbf{v}_{p}=\mathbf{0}
$$

where, in two and three dimensions, $\mathbf{v}_{p}$ contains, respectively, three and six suitably chosen degrees of freedom. The constraint equation is now reduced to

$$
\left[\begin{array}{ll}
\mathbf{C}_{f} & \mathbf{C}_{d}
\end{array}\right]\left[\begin{array}{c}
\mathbf{v}_{f} \\
\mathbf{v}_{d}
\end{array}\right]=\mathbf{0} .
$$

In two dimensions, the above represents three scalar equations involving $2 m-3$ variables, whereas in the three-dimensional case, we have six scalar equations 
and $3 m-6$ variables. Hence, the number of dependent variables - the dimension of $\mathbf{v}_{d}$ and of the square sub-matrix $\mathbf{C}_{d}-$ is 3 and 6 for the two- and three-dimensional cases, respectively. The total number of free variables - the dimension of $\mathbf{v}_{f}$ and number of columns of $\mathbf{C}_{f}-$ is $2 m-6$ and $3 m-12$, respectively in two and three dimensions. Finally, following a trivial manipulation of (45), $\mathbf{v}_{d}$ can be expressed in terms of $\mathbf{v}_{f}$ as

$$
\mathbf{v}_{d}=\mathbf{R} \mathbf{v}_{f}
$$

where

$$
\mathbf{R} \equiv-\mathbf{C}_{d}^{-1} \mathbf{C}_{f}
$$

Note that the dependent degrees of freedom (corresponding to $\mathbf{v}_{d}$ ) must be chosen such that $\mathbf{C}_{d}$ is invertible.

With the above considerations at hand, we can re-define the discrete space (36) of fluctuations and virtual displacements of the RVE as

$$
\tilde{\mathscr{K}}_{\mu}^{h} \equiv\left\{\mathbf{v}=\left[\begin{array}{c}
\mathbf{v}_{i} \\
\mathbf{v}_{f} \\
\mathbf{v}_{d}
\end{array}\right] \quad \mid \mathbf{v}_{d}=\mathbf{R} \mathbf{v}_{f}\right\}
$$

which, for convenience, contains now only the non-prescribed degrees of freedom.

The particularisation of the linearised finite element equation (27) for the present case is obtained, analogously to (33), by splitting the corresponding vectors and tangential stiffness matrix according to the above partitioning and taking (48) into account. This gives

$$
\left\{\left[\begin{array}{c}
\mathbf{F}_{i} \\
\mathbf{F}_{f} \\
\mathbf{F}_{d}
\end{array}\right]^{(k-1)}+\left[\begin{array}{lll}
\mathbf{k}_{i i} & \mathbf{k}_{i f} & \mathbf{k}_{i d} \\
\mathbf{k}_{f i} & \mathbf{k}_{f f} & \mathbf{k}_{f d} \\
\mathbf{k}_{d i} & \mathbf{k}_{d f} & \mathbf{k}_{d d}
\end{array}\right]^{(k-1)}\left[\begin{array}{c}
\delta \tilde{\mathbf{u}}_{i} \\
\delta \tilde{\mathbf{u}}_{f} \\
\mathbf{R} \delta \tilde{\mathbf{u}}_{f}
\end{array}\right]^{(k)}\right\} \cdot\left[\begin{array}{c}
\boldsymbol{\eta}_{i} \\
\boldsymbol{\eta}_{f} \\
\mathbf{R}_{\boldsymbol{\eta}_{f}}
\end{array}\right]=0 \quad \forall \boldsymbol{\eta}_{i}, \boldsymbol{\eta}_{f},
$$

which, after straightforward matrix manipulations taking into account the arbitrariness of $\boldsymbol{\eta}_{i}$ and $\boldsymbol{\eta}_{f}$, is reduced to the final form

$$
\left[\begin{array}{cc}
\mathbf{k}_{i i} & \mathbf{k}_{i f}+\mathbf{k}_{i d} \mathbf{R} \\
\mathbf{k}_{f i}+\mathbf{R}^{T} \mathbf{k}_{d i} & \mathbf{k}_{f f}+\mathbf{k}_{f d} \mathbf{R}+\mathbf{R}^{T} \mathbf{k}_{d f}+\mathbf{R}^{T} \mathbf{k}_{d d} \mathbf{R}
\end{array}\right]^{(k-1)}\left[\begin{array}{c}
\delta \tilde{\mathbf{u}}_{i} \\
\delta \tilde{\mathbf{u}}_{f}
\end{array}\right]^{(k)}=-\left[\begin{array}{c}
\mathbf{F}_{i} \\
\mathbf{F}_{f}+\mathbf{R}^{T} \mathbf{F}_{d}
\end{array}\right]^{(k-1)} .
$$




\section{Assessment of the Gurson porous plasticity model}

In his landmark paper, Gurson (1977) has proposed macroscopic yield surfaces for porous ductile metals by means of a semi-analytical approach relying on the use of the upper bound theorem of plasticity and the analysis of representative cells of material containing a pore embedded in a von Mises type rigid-perfectly plastic matrix. In Gurson's procedure, a sufficient number of points on the corresponding macroscopic yield surface in $p-q$ space $^{2}$ are firstly obtained by computing numerically the overall stress required to cause plastic collapse of the cell under an assumed mechanism. The macroscopic (upper bound) yield surface is then approximated by curve fitting of the resulting points. Under plane strain, the Gurson yield surface has been derived for a cylindrical cell with a single centered cylindrical void and is expressed by means of the yield function

$$
\Phi=q-\left\{\frac{1}{C_{\mathrm{eq}}}\left[1+f^{2}-2 f \cosh \left(\frac{\sqrt{3} p}{\sigma_{Y}}\right)\right]\right\}^{\frac{1}{2}} \sigma_{Y}
$$

were

$$
C_{\mathrm{eq}}=\left(1+3 f+24 f^{6}\right)^{2},
$$

with $f$ denoting the void volume fraction of the cell and $\sigma_{Y}$ the uniaxial yield stress of the matrix material.

In what follows we shall derive alternative predictions of yield functions for porous metals following a methodology similar to that of Gurson. The essential difference between Gurson's approach and present procedure is that, here, the yield surface points will be computed by means of finite element simulations of the plastic collapse of the underlying RVE within the multi-scale constitutive framework described in Sections 2 and 3. That is, each computed point of the macroscopic yield surface point represents the homogenised stress state at which plastic collapse of the RVE occurs under a prescribed monotonically increasing proportional macroscopic strain loading and a particular choice of space $\tilde{\mathscr{K}}_{\mu}^{h}$. This approach has been employed originally by Pellegrino et al. $(1997,1999)$ in the estimation of yield surfaces for periodic elasto-plastic composites where, accordingly, the periodic boundary fluctuations constraint is enforced. In the present study, we shall focus the application on square RVEs under plane strain containing a single centered circular void within an elastic-perfectly plastic von Mises type matrix. In addition to macroscopic yield surfaces predicted under the periodicity constraint, which correspond to periodically perforated media, we shall obtain upper and lower bound surfaces by adopting, respectively, the linear boundary displacements and minimum kinematical constraint assumptions.

\footnotetext{
$\overline{2 p \text { and }} q$ here denote, respectively, the hydrostatic component of the stress tensor and the von Mises equivalent stress.
} 


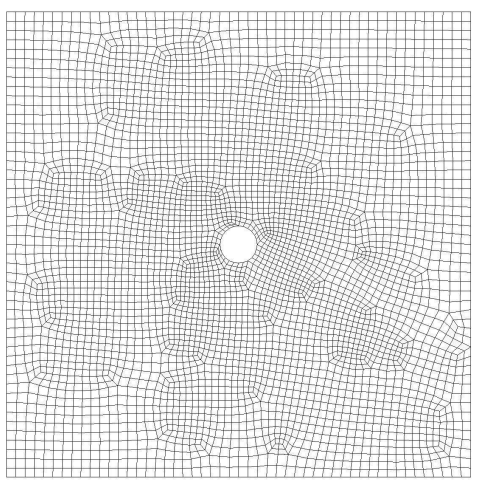

(a) $f=0.5 \%$.

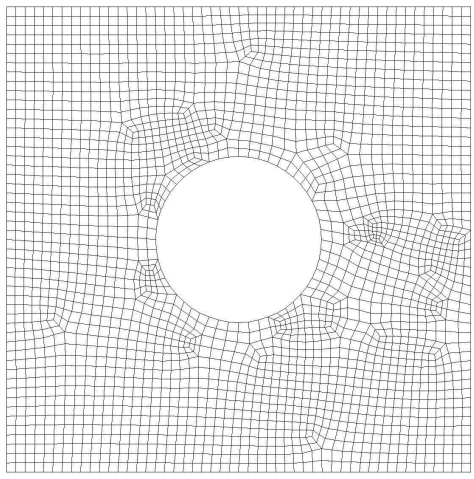

(c) $f=10 \%$.

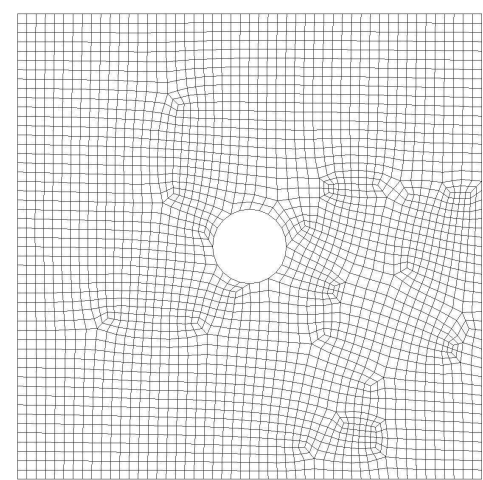

(b) $f=2 \%$.

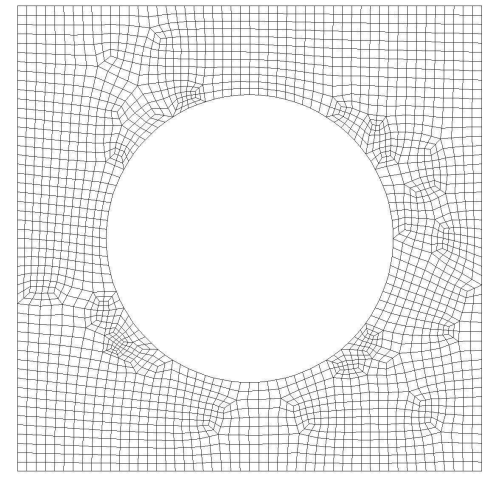

(d) $f=30 \%$.

Fig. 4. RVE geometries and finite element meshes.

\subsection{Computational homogenisation-based methodology}

In order to predict macroscopic yield surfaces as functions of the void ratio of the porous metal, the procedure outlined in the above and further described below will be carried out for RVEs with $f=0.5 \%, 2 \%, 10 \%$ and $30 \%$ - the same void ratios used in Gurson's calculations. The corresponding finite element meshes adopted - all consisting exclusively of isoparametric 8-noded bi-quadratic quadrilaterals with (reduced) $2 \times 2$-point Gaussian integration quadrature - are illustrated in Figure 4. The material properties chosen for the von Mises matrix are the following: Young's modulus $E=200 \mathrm{GPa}$, Poisson's ratio $\nu=0.3$ and yield stress $\sigma_{Y}=0.24 \mathrm{GPa}$.

The loading programme imposed on the RVEs consists in prescribing a macroscopic strain path

$$
\varepsilon(\gamma)=\gamma \bar{\varepsilon}
$$

parametrised by a monotonically increasing load factor $\gamma$, where $\bar{\varepsilon}$ is the unit 
plane strain tensor defined as

$$
\bar{\varepsilon}=\alpha\left[\begin{array}{cc}
\frac{1}{\sqrt{2}} & 0 \\
0 & \frac{1}{\sqrt{2}}
\end{array}\right]+\sqrt{1-\alpha^{2}}\left[\begin{array}{cc}
0 & \frac{1}{\sqrt{2}} \\
\frac{1}{\sqrt{2}} & 0
\end{array}\right]
$$

satisfying

$$
\|\bar{\varepsilon}\|=1,
$$

and $\alpha \in[0,1]$ is a prescribed parameter defining the direction of $\varepsilon$ in strain space. Note that $\alpha=0$ corresponds to a pure shear direction whereas $\alpha=1$ is the in-plane spherical direction. Any other value of $\alpha$ within this range is a combination of both.

For each chosen value of $f$, finite element simulations are carried out for a number of values of $\alpha$ (or directions in strain space) covering the range [0,1]. For each value of $\alpha$, the load factor $\gamma$ is increased monotonically, starting from an unstressed plastically virgin state of the RVE with $\gamma=0$, until plastic collapse of the RVE occurs. The corresponding macroscopic collapse stress is computed according to (9) as the volume average of the finite element-predicted microscopic stress field $\boldsymbol{\sigma}_{\mu}$ at the collapse state of the RVE. The hydrostatic and von Mises components of the macroscopic collapse stress define a yield surface point. Finally, an estimate of the functional format of the $p-q$ space yield surface is obtained by curve fitting of the yield surface points obtained by this procedure.

\subsection{Estimated yield surfaces}

The macroscopic yield surface points obtained according to the above methodology are plotted in Figure 5 in the normalised $\bar{p}-\bar{q}$ space, where

$$
\bar{p} \equiv \frac{p}{\sigma_{Y}} \quad \text { and } \quad \bar{q} \equiv \frac{q}{\sigma_{Y}} .
$$

The results are shown for the four values of void ratio considered. To describe the corresponding approximate yield surfaces which fit the computed points we propose the following alternative to the original plane strain Gurson yield function (51):

$\bar{\Phi}=q-C_{1}\left[1-C_{2} \sin ^{2}\left(\frac{\pi p}{2 P_{m}}\right)\right]\left\{\frac{1}{C_{\mathrm{eq}}}\left[1+f^{2}-2 f C_{3} \cosh \left(\frac{\sqrt{3} C_{4} p}{\sigma_{Y}}\right)\right]\right\}^{\frac{1}{2}} \sigma_{Y}$,

where $P_{m}$ is the value of $p$ at failure obtained with $\alpha=1$ and $C_{i}, i=1, \cdots, 4$, are non-dimensional parameters determined so as to provide a best fit to the yield surface point data. The resulting approximate yield surfaces in $\bar{p}-\bar{q}$ space 
(a)

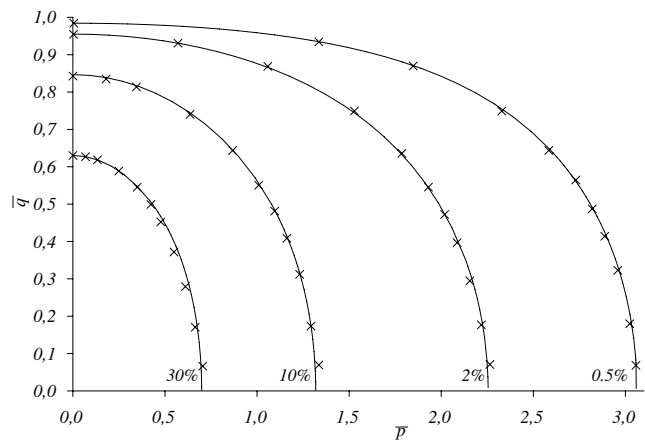

(c)

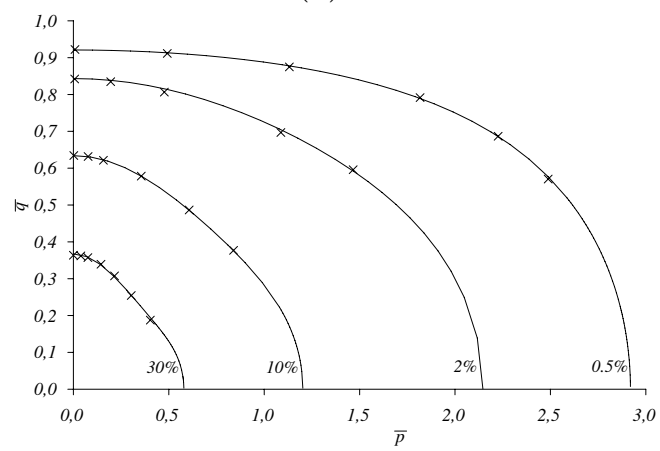

(b)

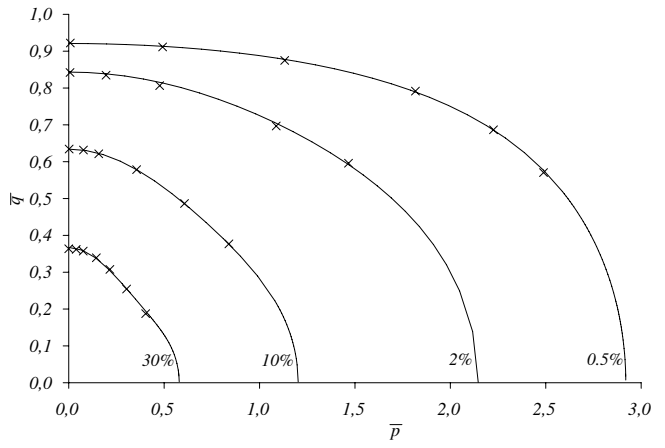

Fig. 5. Estimated yield surfaces for $f=0.5 \%, 2 \%, 10 \%$ and 30\%. Computed points and approximate functional form. (a) Linear boundary displacements; (b) Periodic boundary fluctuations; and (c) Minimum kinematical constraint (uniform boundary tractions).

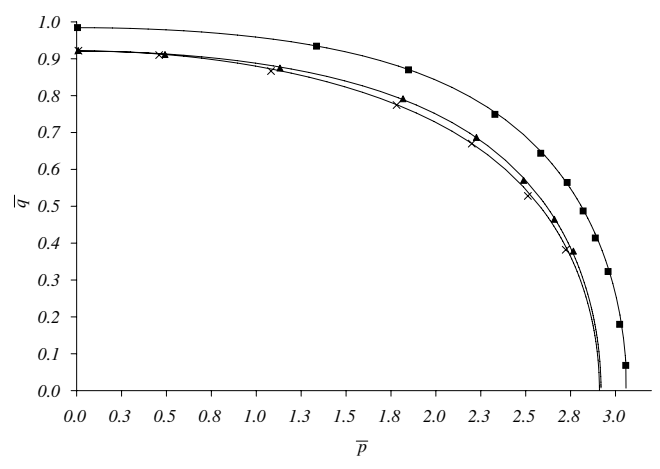

(a) $f=0.5 \%$.

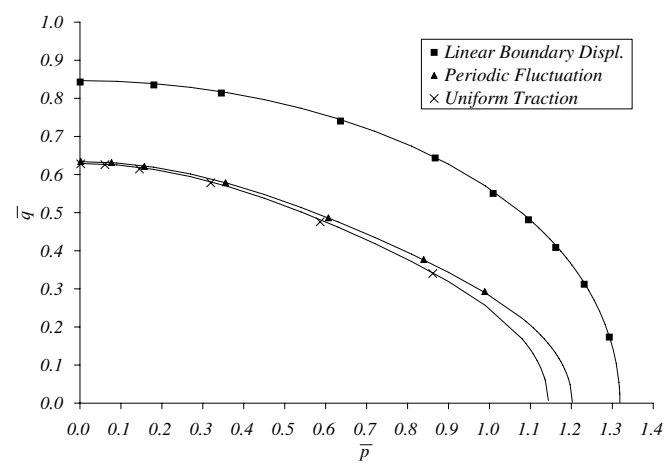

(b) $f=10 \%$.

Fig. 6. Estimated yield surfaces under different RVE kinematical constraints.

are also shown in Figure 5. For ease of comparison, the results for the three RVE kinematical constraints considered are re-grouped in Figures 6(a) and (b) for $f=0.5 \%$ and $10 \%$, respectively. As expected, the linear boundary displacements condition (the most constraining of the three kinematical assumptions considered) provides an upper bound for the yield locus of the porous metal. The minimum kinematical constraint, in turn, provides a lower bound. The results under the periodicity assumption lie between the other two in very 


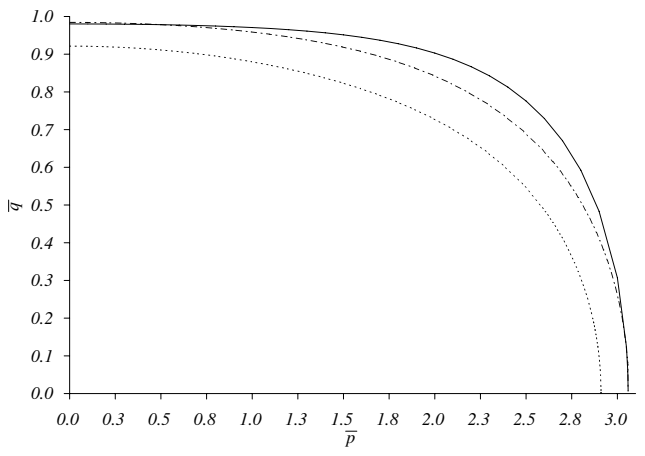

(a) $f=0.5 \%$.

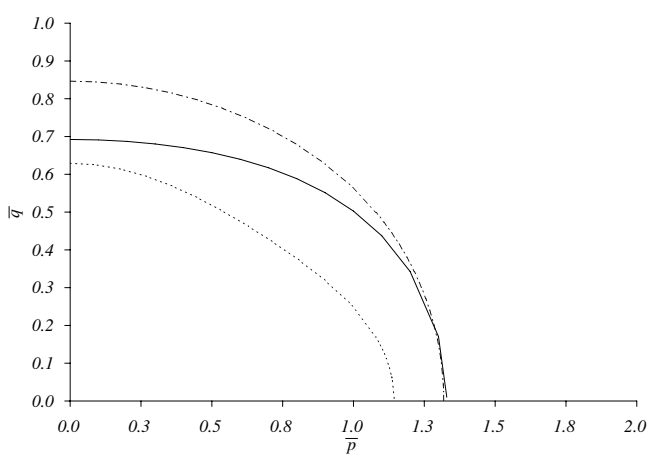

(c) $f=10 \%$.

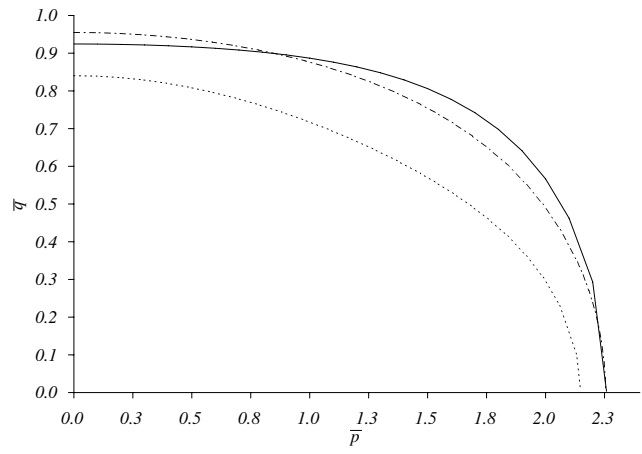

(b) $f=2 \%$.

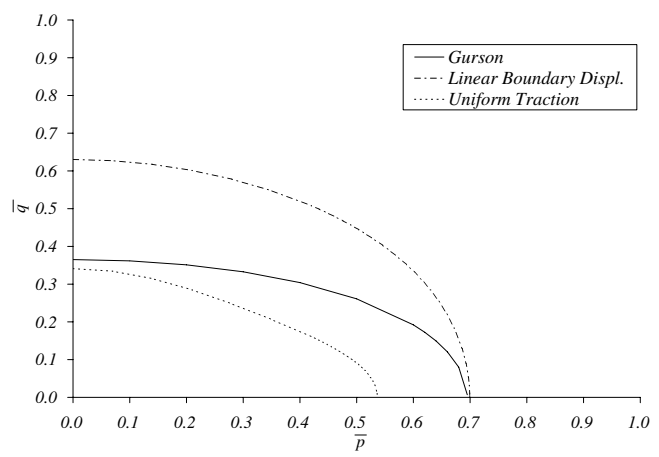

(d) $f=30 \%$.

Fig. 7. Gurson's criterion and computed lower and upper bounds.

close proximity to the lower bound.

In Figure 7 the upper and lower bounds are plotted along the original plane strain Gurson yield surface. The approximate surface obtained under the periodicity assumption is omitted due to its proximity to the lower bound. It should be noted that the present upper and lower bounds lie farther apart for higher void ratios. For higher void ratios, the Gurson surface lies between the present upper and lower bounds - closer to the upper bound for states near pure hydrostatic stress and closer to the lower bound for states close to pure shear. For lower void ratios the Gurson surface predicts, for some combinations of hydrostatic and von Mises equivalent stress, a yield limit above the present upper bound. Under such conditions, the Gurson criterion is expected to overestimate the yield strength of the porous metal.

\subsection{General functional forms}

The values of the $C_{i}$ parameters of the proposed yield function (57), obtained for the estimated yield surfaces presented in the above, are given in Table 1. In order to obtain general functional forms of yield function for each type of assumed kinematical constraint under a range of void ratios, we carry out one 


\begin{tabular}{|c||c|c|c||c|c|c||c|c|c||c|c|c|}
\hline \multicolumn{1}{|c||}{$f$} & \multicolumn{3}{c||}{$0.5 \%$} & \multicolumn{3}{c||}{$2 \%$} & \multicolumn{3}{c||}{$10 \%$} & \multicolumn{3}{c|}{$30 \%$} \\
\hline & lin. & per. & min. & lin. & per. & min. & lin. & per. & min. & lin. & per. & min. \\
\hline$C_{1}$ & 1.04 & 0.98 & 0.98 & 1.17 & 1.055 & 1.055 & 1.58 & 1.235 & 1.23 & 2.05 & 1.295 & 1.26 \\
$C_{2}$ & 0.005 & 0.02 & 0.06 & 0.0188 & 0.128 & 0.145 & 0.035 & 0.255 & 0.2415 & 0.0459 & 0.345 & 0.307 \\
$C_{3}$ & 6.776 & 8.034 & 8.08 & 5.52 & 6.45 & 6.45 & 2.055 & 2.30 & 2.32 & 0.6344 & 0.81 & 0.90 \\
$C_{4}$ & 0.6347 & 0.63 & 0.635 & 0.55 & 0.535 & 0.535 & 0.61 & 0.615 & 0.645 & 0.99 & 1.01 & 1.00 \\
\hline
\end{tabular}

Table 1

Parameters $C_{1}, \cdots, C_{4}$ for alternative (57) to Gurson's yield function. Linear RVE boundary displacements, periodic fluctuations and minimum kinematical constraint.

last curve fitting exercise based on the data of Table 1 to express the coefficients $C_{i}$ as functions of $f$. Upon close inspection of the data, we find that each coefficient varies as a function of $f$ following trends that do not depend on the assumed kinematical constraint. The functional forms found to be particularly suitable to express $C_{1}, C_{2}, C_{3}$ and $C_{4}$ as functions of $f$ are, respectively, as a fourth order polynomial, a logarithmic function, an exponential function and a fifth order polynomial. Under the assumption of linear RVE boundary displacements, for which an upper bound for the homogenised yield surface is obtained, the following functional form for the coefficients $C_{i}$ is determined:

$$
\begin{aligned}
& C_{1}(f)=-435.6 f^{4}+277.9 f^{3}-66.4 f^{2}+10.2 f+0.99 \\
& C_{2}(f)=0.01 \ln (0.015 f)+0.1 \\
& C_{3}(f)=6.7 \exp (-15 f)+0.56 \\
& C_{4}(f)=-2604 f^{5}+2524 f^{4}-921.2 f^{3}+153 f^{2}-9 f+0.6761 .
\end{aligned}
$$

Under the assumption of periodicity of displacement fluctuations, we have:

$$
\begin{aligned}
& C_{1}(f)=-256.2 f^{4}+183.3 f^{3}-48.5 f^{2}+6.12 f+0.95 \\
& C_{2}(f)=0.08 \ln (0.01 f)+0.81 \\
& C_{3}(f)=7.9 \exp (-16.5 f)+0.76 \\
& C_{4}(f)=-3232 f^{5}+3095.6 f^{4}-1111 f^{3}+180 f^{2}-10.3 f+0.677,
\end{aligned}
$$

and, finally, for the minimum kinematical constraint (or uniform RVE boundary traction), which provides a lower bound yield surface, we obtain:

$$
\begin{aligned}
& C_{1}(f)=-223.6 f^{4}+170.7 f^{3}-48 f^{2}+6.11 f+0.95 \\
& C_{2}(f)=0.06 \ln (0.03 f)+0.59 \\
& C_{3}(f)=7.9 \exp (-16.5 f)+0.82 \\
& C_{4}(f)=-3813 f^{5}+3588 f^{4}-1264.6 f^{3}+199.7 f^{2}-11 f+0.6853 .
\end{aligned}
$$




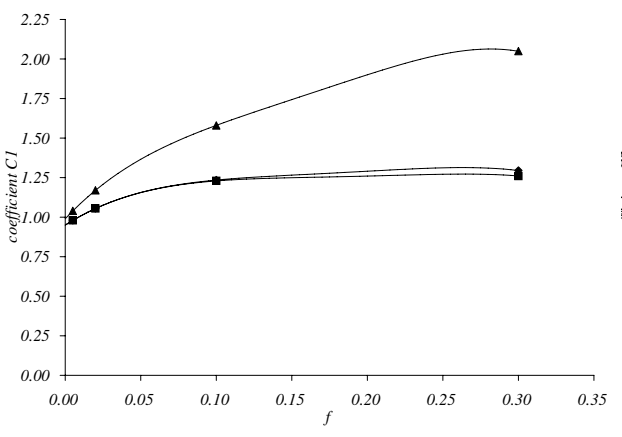

(a) $C_{1}$.

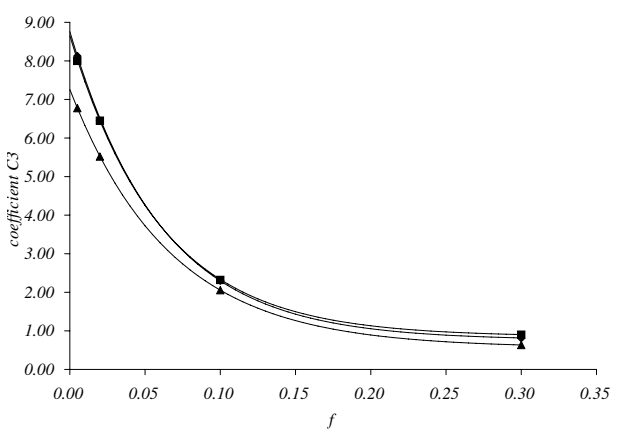

(c) $C_{3}$.

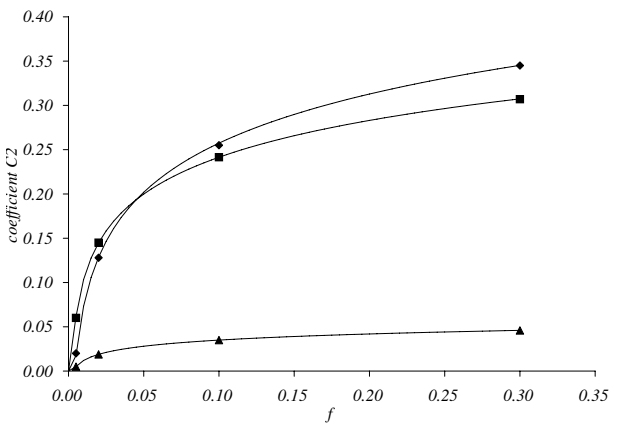

(b) $C_{2}$.

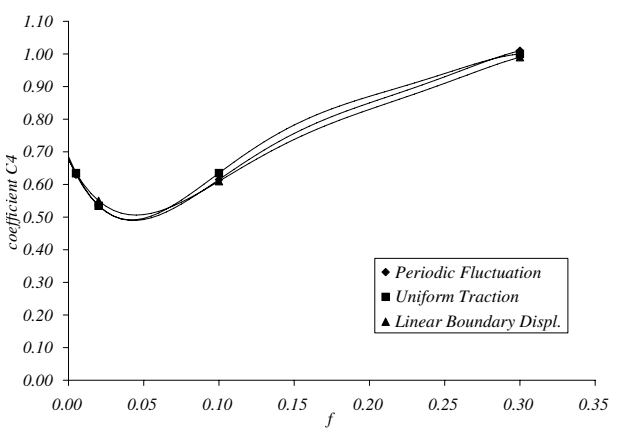

(d) $C_{4}$.

Fig. 8. Estimated coefficients for expression $\bar{\Phi}$.

The above functions together with the data of Table 1 are plotted in Figure 8 .

\subsection{Mechanisms of plastic collapse}

The differences in macroscopic yield strength observed under the assumed RVE kinematic constraints considered arise from the fact that distinct kinematical constraints lead in general to different mechanisms being responsible for the plastic collapse of the RVE. This is illustrated Figures 9 and 10. These show contour plots of incremental effective plastic strain of the RVE obtained at collapse states in the finite element solution for $\alpha=0$ (pure shear strain path) and $\alpha=1$ (spherical in-plane strain path), respectively. In both cases the void ratio is $f=10 \%$ and the three kinematical constraints are considered. It is clear that the collapse mechanism triggered under the linear boundary displacements constraint is quite different from the mechanisms under the other two constraints considered This explains the yield strength differences observed previously. Also, it should be noted that the mechanisms associated with collapse under the periodic fluctuations and minimum constraint assumptions are very similar to each other, justifying the similarity between the homogenised yield surfaces obtained under these two conditions. 


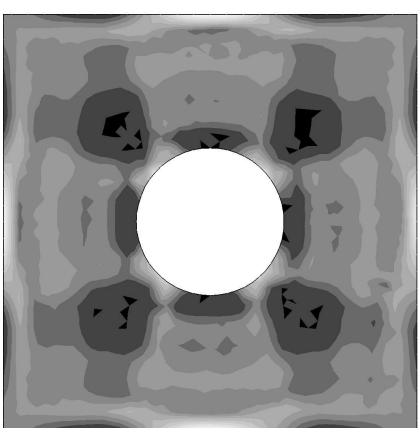

(a) Linear boundary displacements.

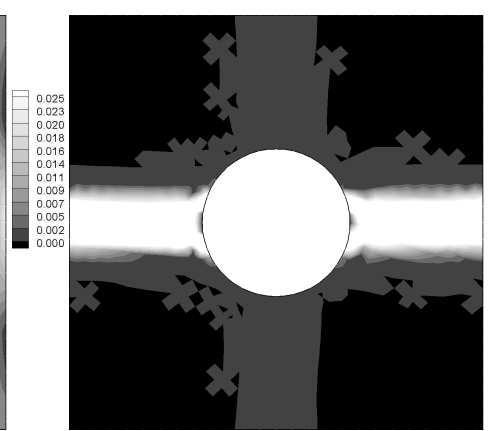

(b) Periodic fluctuation.

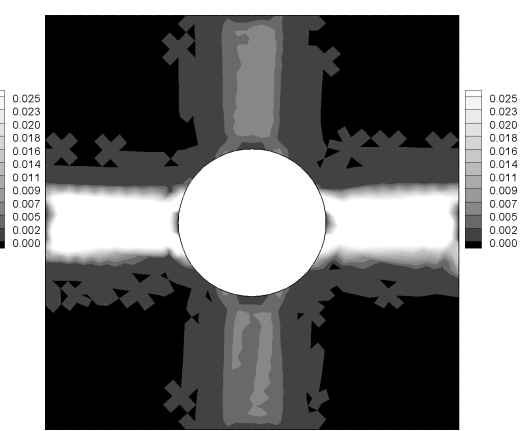

(c) Minimum constraint.

Fig. 9. RVE collapse for $f=10 \%$ and $\alpha=0$. Contour plots of incremental effective plastic strain.

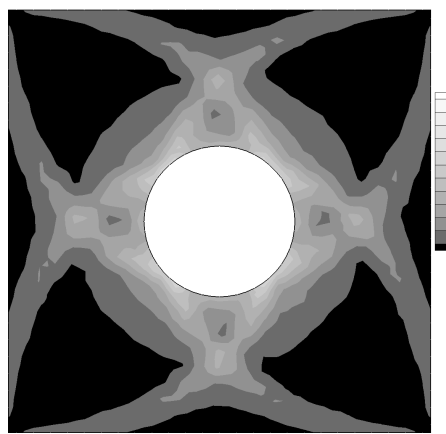

(a) Linear boundary displacements.

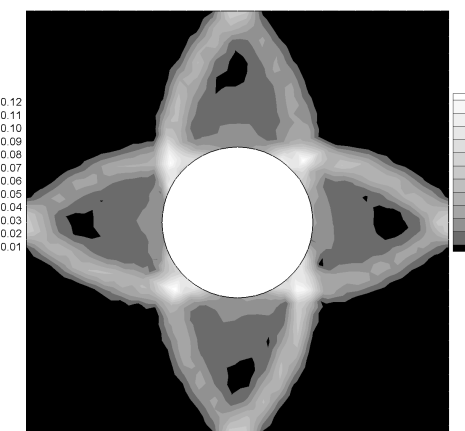

(b) Periodic fluctuation.

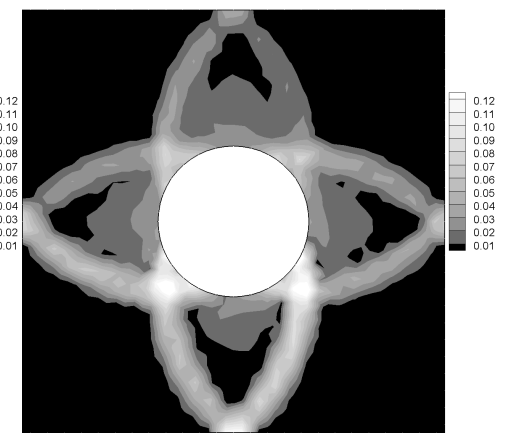

(c) Minimum constraint.

Fig. 10. RVE collapse for $f=10 \%$ and $\alpha=1$. Contour plots of incremental effective plastic strain.

\section{Concluding remarks}

Yield surface estimates for porous metals have been obtained by means of a purely kinematical finite element-based multi-scale constitutive modelling approach relying on the volume averaging of the strain and stress tensors over a representative volume element of material. The macroscopic yield surface estimates have been obtained under three different kinematical constraints of the RVE: linear boundary displacements (an upper bound); periodic displacement fluctuations (corresponding to periodically perforated media); and, minimum kinematical constraint or uniform boundary traction (a lower bound). An assessment of the classical Gurson criterion has been carried out in the light of the computed bounds. A modification of the Gurson yield function has been proposed which is able to capture the yield loci of porous metals under the three kinematical assumptions considered. The proposed functional form can be used in the estimation of bounds for the plastic behaviour of porous metals under plane strain. 


\section{Acknowledgements}

P.J. Blanco and E.A. de Souza Neto were partly supported by CNPq (Brazilian Research Council) under the grants 140686/2005-3 and 381942/2004-1, respectively. S.M. Giusti was supported by CAPES (Brazilian Higher Education Staff Training Agency). This support is gratefully acknowledged.

\section{References}

de Souza Neto, E.A. \& Feijóo, R.A. (2006) Variational foundations of multiscale constitutive models of solid: Small and large strain kinematical formulation, LNCC Research \& Development Report, No. 16/2006, National Laboratory for Scientific Computing, Petrópolis, Brazil.

Gărăjeu, M. \& Suquet, P. (1997) Effective properties of porous ideally plastic or viscoplastic materials containing rigid particles, J. Mech. Phys. Solids, 45(6):873-902.

Gurson, A.L. (1977) Continuum theory of ductile rupture by void nucleation and growth: Part I-yield criteria and flow rules for porous ductile media, $J$. Engng. Mat. Tech., Transactions of the ASME.

Hill, R. (1963) Elastic properties of reinforced solids: Some theoretical principles, J. Mech. Phys. Solids, 11:357-372.

Hill, R. (1965) A self-consistent mechanics of composite materials, J. Mech. Phys. Solids, 13(4):213-222.

Kouznetsova, V., Geers, M.G.D. \& Brekelmans, V.A.M. (2002) Multiscale constitutive modelling of heterogeneous materials with a gradientenhanced computational homogenization scheme, Int. J. Num. Meth. Engng., 54:1235-1260.

Mandel, J. (1971) Plasticité Classique at Viscoplasticité, CISM Lecture Notes, Springer-Verlag, Udine, Italy.

Michel, J.C. \& Suquet, P. (1992) The constitutive law of nonlinear viscous and porous materials, J. Mech. Phys. Solids, 40(4):783-812.

Michel, J.C., Moulinec, H. \& Suquet, P. (1999) Effective properties of composite materials with periodic microstructure: a computational approach, Comp. Meth. Appl. Mech. Engng., 172:109-143.

Michel, J.C., Moulinec, H. \& Suquet, P. (2001) A computational scheme for linear and non-linear composites with arbitrary phase contrast, Int. J. Numer. Meth. Engng., 52:139-160.

Miehe, C., Schotte, J. \& Schröder, J. (1999) Computational Micro-Macro Transitions and Overall Moduli in the Analysis of Polycrystals at Large Strains, Comput. Materials Sci., 16:372-382.

Miehe, C., Shotte, J. \& Lambrecht, M. (2002) Homogenization of Inelastic Solid Materials at Finite Strains Based on Incremental Minimization Prin- 
ciples. Application to the Texture Analysis of Polycrystals, J. Mech. Phys. Solids, 50(10):2123-2167.

Miehe, C., \& Koch, A. (2002) Computational micro-macro transitions of discretized microstructures undergoing small strains, Arch. Appl. Mech., 72:300-317.

Pellegrino, C., Galvanetto, U. \& Schrefler, B.A. (1997) Computational techniques for periodic composite materials with elasto-plastic components, In Computational Plasticity: Fundamentals and Applications, D.R.J.Owen, E.Oñate and E.Hinton (eds.), p 1229-1236, CIMNE, Barcelona.

Pellegrino, C., Galvanetto, U. \& Schrefler, B.A. (1999) Numerical homogenisation of periodic composite materials with non-linear material components, Int. J. Numer. Meth. Engng., 46:1609-1637.

Simo, J.C. \& Hughes, T.J.R (1998) Computational Inelasticity, SpringerVerlag, New York.

Swan, C.C. (1994) Techniques for stress- and strain-controlled homogenization of inelastic periodic composites, Comp. Meth. Appl. Mech. Engng., 117:249267.

Terada, K., Saiki, I., Matsui. K. \& Yamakawa, Y. (2003) Two-scale kinematics and linearization for simultaneous two-scale analysis of periodic heterogeneous solids at finite strains, Comp. Meth. Appl. Mech. Engng., 192:35313563.

Tvergaard, V. (1981) Influence of voids on shear band instabilities under plane strain conditions, Int. J. Fracture, 17:389-407. 